\title{
Spontaneous emulsification induced by nanoparticle surfactants $\odot \odot$
}

Cite as: J. Chem. Phys. 153, 224705 (2020); https://doi.org/10.1063/5.0029016

Submitted: 08 September 2020 . Accepted: 15 November 2020 . Published Online: 14 December 2020

(iD J. Hasnain, (D) Y. Jiang, (D) H. Hou, J. Yan, (D) L. Athanasopoulou, (D) J. Forth, (D) P. D. Ashby, (D) B. A. Helms, T. P. Russell, and (D) P. L. Geissler

\section{COLLECTIONS}

This paper was selected as Featured

SCI) This paper was selected as Scilight
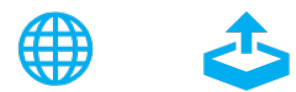

\section{ARTICLES YOU MAY BE INTERESTED IN}

\section{Reflections on electron transfer theory}

The Journal of Chemical Physics 153, 210401 (2020); https://doi.org/10.1063/5.0035434

Vibronic resonance is inadequately described by one-particle basis sets

The Journal of Chemical Physics 153, 224114 (2020); https://doi.org/10.1063/5.0029027

Confronting pitfalls of Al-augmented molecular dynamics using statistical physics

The Journal of Chemical Physics 153, 234118 (2020); https://doi.org/10.1063/5.0030931

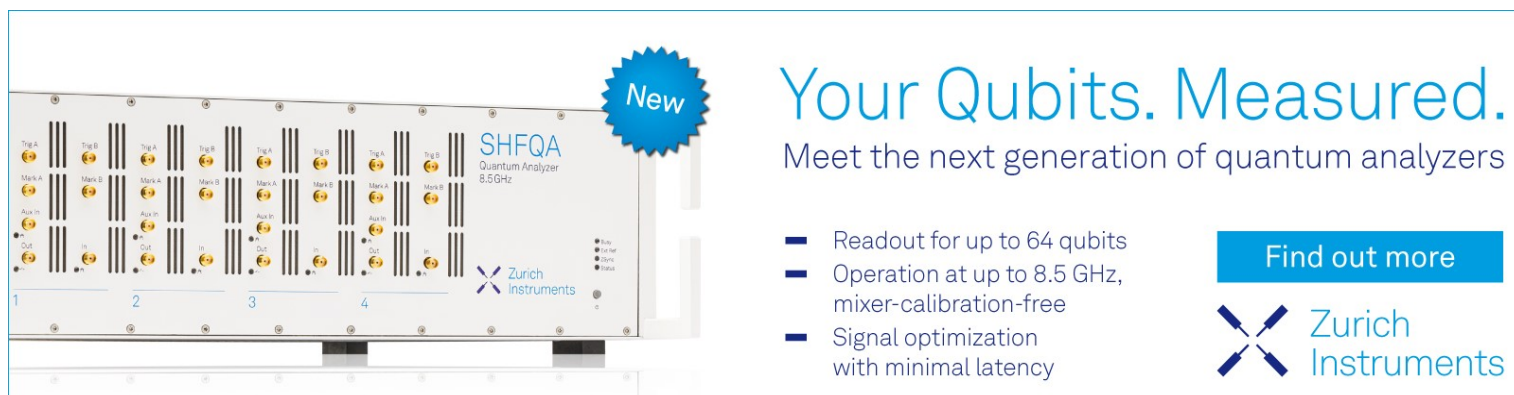




\title{
Spontaneous emulsification induced by nanoparticle surfactants $\mathrm{F}$ ( 61
}

\author{
Cite as: J. Chem. Phys. 153, 224705 (2020); doi: 10.1063/5.0029016 \\ Submitted: 8 September 2020 - Accepted: 15 November 2020 • \\ Published Online: 14 December 2020
}

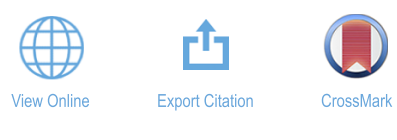

\author{
J. Hasnain, ' (D) Y. Jiang,' (D) H. Hou, ' (D) J. Yan, ' L. Athanasopoulou, ${ }^{2}$ (D) J. Forth, ' (D) P. D. Ashby, \\ B. A. Helms, ${ }^{1}$ (D) T. P. Russell, ${ }^{1}$ and P. L. Geissler ${ }^{3, a)}$ (D)
}

\begin{abstract}
AFFILIATIONS
${ }^{1}$ Materials Sciences Division, Lawrence Berkeley National Laboratory, Berkeley, California 94720, USA

${ }^{2}$ Faculty of Mathematics and Physics, University of Ljubjana, Jadranska 19, SI-1000 Ljubjana, Slovenia

${ }^{3}$ Department of Chemistry, University of California Berkeley, Berkeley, California 94720, USA
\end{abstract}

a) Author to whom correspondence should be addressed: geissler@berkeley.edu

\begin{abstract}
Microemulsions, mixtures of oil, water, and surfactant, are thermodynamically stable. Unlike conventional emulsions, microemulsions form spontaneously, have a monodisperse droplet size that can be controlled by adjusting the surfactant concentration, and do not degrade with time. To make microemulsions, a judicious choice of surfactant molecules must be made, which significantly limits their potential use. Nanoparticle surfactants, on the other hand, are a promising alternative because the surface chemistry needed to make them bind to a liquid-liquid interface is both well flexible and understood. Here, we derive a thermodynamic model predicting the conditions in which nanoparticle surfactants drive spontaneous emulsification that agrees quantitatively with experiments using Noria nanoparticles. This new class of microemulsions inherits the mechanical, chemical, and optical properties of the nanoparticles used to form them, leading to novel applications.
\end{abstract}

Published under license by AIP Publishing. https://doi.org/10.1063/5.0029016

\section{INTRODUCTION}

Interfacial tension (IFT), the free energy penalty of exposing one immiscible liquid to another, is the greatest impediment to the stability of emulsions since it drives them to phase separate. To counteract this, emulsions can be stabilized by introducing amphiphilic particles or molecules that reduce the interfacial tension by segregating to the liquid-liquid interface. To this end, interfacially active colloidal particles enhance the mechanical stability of an emulsion, but high-energy processing is usually necessary to form such Pickering emulsions. In contrast, molecular surfactants can generate a thermodynamically stable emulsion, at no energetic cost; ${ }^{2-7}$ however, their influence is very sensitive to chemical composition, severely limiting the scope and customizability of this approach. Here, we show that interfacially active nanoparticle surfactants can drive spontaneous emulsification, provided that they are sufficiently small and interfacially active. Using thermodynamic arguments, we formulate a design strategy that is realized using $1 \mathrm{~nm}$ organic cages with high surface-charge density that are capable of spontaneously creating aqueous droplets in a silicone oil bath. Furthermore, a few simple pendant drop measurements allow us to parameterize our model, leading to quantitative correspondence with more sophisticated experiments. Due to the generality of our arguments, these considerations form the basis for a new class of particle-based microemulsions with controllable surface area, functionality, and thermodynamic stability. Such microemulsions can be used to efficiently formulate pharmaceuticals ${ }^{8}$ and biodegradable pesticides, ${ }^{9}$ carry out nanoparticle synthesis, ${ }^{10}$ and perform catalytic transformations.

Particle-based microemulsions are a promising means of selfassembling an emulsion of monodisperse microscopic droplets, whose surfaces inherit the plasmonic, ${ }^{12}$ magnetic, ${ }^{13}$ or mechanical ${ }^{14}$ properties of a growing family of interfacially active nanoparticles. ${ }^{15}$ Despite the plethora of interfacially active particles, including some 
capable of stabilizing bicontinuous liquid interfaces, ${ }^{16,17}$ particleinduced spontaneous emulsification has only been demonstrated for specific oils, ${ }^{18-21}$ and theoretical efforts have mainly focused on the thermodynamics of molecular microemulsifiers ${ }^{2-30}$ that lack the functionality of nanoparticles. The aim of this work is to determine the conditions under which nano-scale particles induce spontaneous emulsification.

\section{RESULTS}

\section{A. Theoretical considerations}

To describe nanoparticle adsorption to an oil-water interface and its effect on the interfacial tension (IFT), we formulated a thermodynamic model of an aqueous droplet containing interfacially active nanoparticles that is suspended in an oil bath. Our microscopic model treats the oil-water interface as a two-dimensional plane onto which nanoparticles with the radius $r$ adsorb with the binding energy $U$ and packing fraction $\eta$. The interior of the droplet is a three-dimensional volume $V$ containing a volume fraction $\phi$ of non-adsorbed nanoparticles. The binding energy of the nanoparticles, $U$, is a function of the type of oil used, the $\mathrm{pH}$ of the aqueous phase, and the charge distribution at the liquidliquid interface along with its interaction with the nanoparticle surface.

The system reaches equilibrium, when the thermodynamic drive for particle adsorption is canceled by the thermodynamic drive for desorption [green arrows in Fig. 1(a)]. From this condition, we derive

$$
\mu_{2 \mathrm{~d}}(\eta)-U-k_{\mathrm{B}} T \ln \frac{2 r}{\Lambda}=\mu_{3 \mathrm{~d}}(\phi)
$$

where $\mu_{3 \mathrm{~d}}$ is the chemical potential of the nanoparticles in the bulk aqueous phase, $k_{\mathrm{B}}$ is the Boltzmann constant, $T$ is the temperature, and $\Lambda$ is the thermal de Broglie wavelength. For the interfacial population, Eq. (1) separates contributions from the binding energy, $U$, fluctuations perpendicular to the interface, $k_{\mathrm{B}} T \ln 2 r \Lambda^{-1}$, and the chemical potential of nanoparticles strictly confined to a two-dimensional plane, $\mu_{2 \mathrm{~d}}(\eta)$, which is a function of the packing fraction at the interface, $\eta$ (a detailed derivation is provided in the supplementary material).

The interfacial tension (IFT) of the oil-water interface is the difference between the surface tension in the absence of adsorbed nanoparticles, $\gamma$, and the in-plane pressure exerted by the adsorbed particles [red arrows in Fig. 1(a)],

$$
\mathrm{IFT}=\gamma-k_{\mathrm{B}} T \frac{\eta Z(\eta)}{\pi r^{2}}
$$

where $Z(\eta)$ is the two-dimensional compressibility of the adsorbed particles. In this framework, spontaneous emulsification occurs when the packing fraction of particles at the oil-water interface is so large that the free energy penalty for the increasing interfacial area, IFT, vanishes.

For this model to make quantitative predictions, it is necessary to specify an equation of state for the nanoparticles. The equation of state relates the pressure of an ensemble of particles to their density and temperature, and its functional form depends crucially on the interparticle interactions. Once an appropriate equation of state has been selected, the chemical potential follows from the Gibbs-Duhem relation. Although the nanoparticle interactions are unknown, sensible choices for the equation of state can still be made. It has been shown that the phase behavior of particles interacting via sufficiently short-ranged repulsion can be mapped to the hard sphere
A

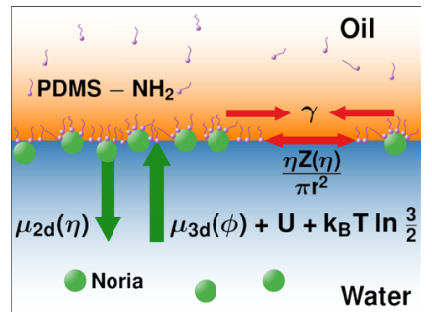

C

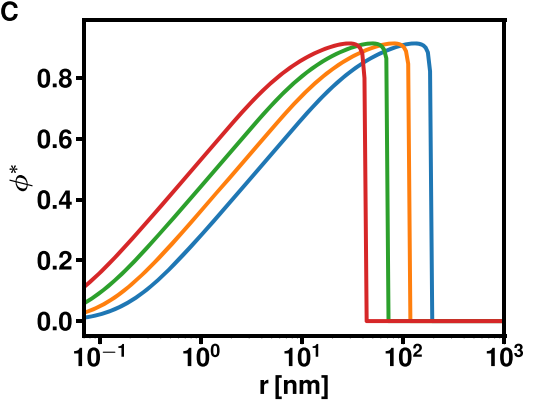

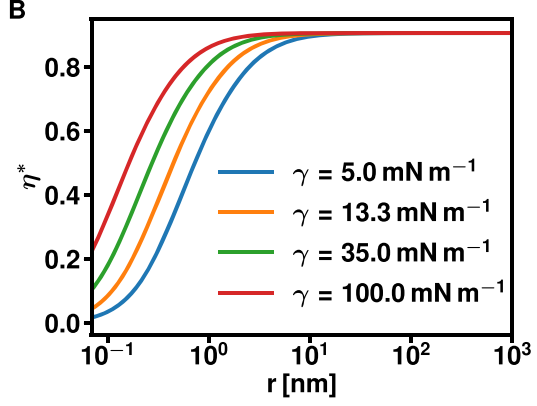

D

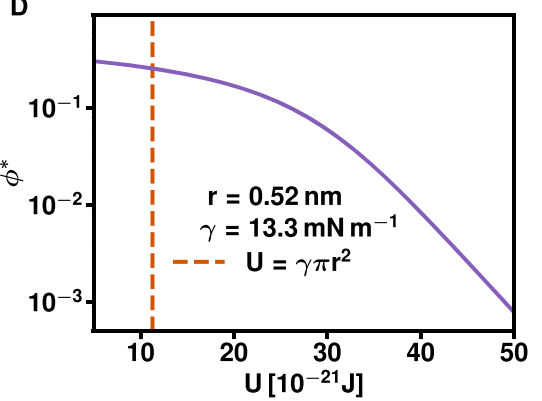

FIG. 1. Thermodynamics of spontaneous emulsification. (a) Schematic illustration of Noria nanoparticle surfactants and of Eqs. (1) and (2). (b) Critical packing fraction necessary to form a microemulsion, $\eta^{*}$, as a function of particle radius, $r$, for a variety of interfacial tensions, $\gamma$. The orange curve for $\gamma=13.3 \mathrm{mN} \mathrm{m}^{-1}$ corresponds to our experiments. (c) Critical volume fraction of the nanoparticles, $\phi^{*}$, assuming that the binding energy scales as $U=\gamma \pi r^{2}$ [colors as in (b)]. (d) Critical volume fraction, $\phi^{*}$, as a function of the binding energy, $U$, for the interfacial tension and nanoparticle radius corresponding to our experiments. 
equation of state using the second virial coefficient. ${ }^{31,32}$ Alternatively, if it is suspected that attractive interactions are significant, then Weeks-Chandler-Anderson theory prescribes that the addition of a mean field attraction term to the hard sphere equation of state accurately characterizes the system. ${ }^{33}$ In this study, we find quantitative correspondence with experimental data by treating our nanoparticles as hard spheres and hard disks with an effective particle radius $r$ and binding strength $U$ that are fit onto the data. In the supplementary material, we consider interparticle attractions at a mean-field level and find them to be incompatible with experimental observations.

Of the many approximations available, we use the chemical potential, $\mu_{2 \mathrm{~d}}(\eta)$, and compressibility, $Z(\eta)$, for hard disks developed by Santos et. al. ${ }^{34}$ for the particles at the interface. These expressions of the chemical potential and the compressibility are monotonically increasing functions of the packing fraction that diverge at densest circle packing, reproduce the second virial coefficient exactly, and accurately approximate numerical results. ${ }^{34}$ Since the volume fraction of the unbound nanoparticles is well below 0.55 , we use the Carnahan-Starling hard sphere approximation for the chemical potential, $\mu_{3 \mathrm{~d}}(\phi)$.

The packing fraction at which spontaneous emulsification occurs, $\eta^{*}$, for typical interfacial tensions, $\gamma$, is obtained numerically from Eq. (2) and increases monotonically with the particle radius, $r$ [Fig. 1(b)]. The model predicts that for nanoparticles larger than $10 \mathrm{~nm}$, nearly densest packing at the interface is necessary to create a microemulsion.

Next, we insert $\eta^{*}$ into Eq. (1) and assume that $U$ is entirely due to the screening of the oil phase from the water phase, $U=\gamma \pi r^{2}$. This expression of the binding energy assumes that the nanoparticles have no preference for either the oil or the water phase and constitutes the best-case scenario for microemulsification using the conventional understanding of nanoparticle adsorption. ${ }^{35}$ We find that nanoparticles in the $0.5 \mathrm{~nm}-20 \mathrm{~nm}$ range require threshold volume fractions, $\phi^{*}$, which are so large that they are likely to precipitate from solution [Fig. 1(c)]. Nanoparticles with diameters on the order of $100 \mathrm{~nm}$, on the other hand, have very low critical volume fractions due to binding energies that exceed thousands of $k_{\mathrm{B}} T=\beta^{-1}$. A naive analysis, therefore, encourages the use of microemulsifiers that are as large as possible. This strategy is unwise for three reasons: To overwhelm the interfacial tension, large particles need to attain very high packing fractions at the interface [Fig. 1(b)], which can be prohibited by the kinetic and energetic barriers associated with inserting particles into densely packed monolayers. ${ }^{36}$ Furthermore, binding energies on the order of thousands of $k_{\mathrm{B}} T$ are so large that nanoparticles adsorb irreversibly to the interface, which generates deep kinetic traps en route to macroscopic mixing. ${ }^{36}$ Finally, the process of spontaneous emulsification involves distortion of a flat interface sufficient to create buds that detach from a mother droplet and escape into the bulk [Fig. 2(d)]. For large particles, these buds correspond to extremely rare fluctuations in interfacial shape. The resulting dynamical bottleneck can be overcome by mechanical agitation, as in the formation of particle-stabilized Pickering emulsions, but this comes at high energetic cost. ${ }^{1}$ We conclude that spontaneous emulsification is kinetically accessible only for sufficiently small particles, but the required solution concentrations, $\phi^{*}$, of small particles are difficult to realize in practice. For this reason, few successful nanometer-scale microemulsifiers have been identified to date.

One way to reduce $\phi^{*}$ would be to increase the binding energy of the nanoparticles, $U$, beyond what is expected from reducing the area of contact between the oil and water phases [Fig. 1(d)]. Alternatively, adsorbed particles could have substantial attractive interactions. Both mechanisms are likely to be important for molecular surfactants but are challenging to engineer for synthetic nanoparticles.

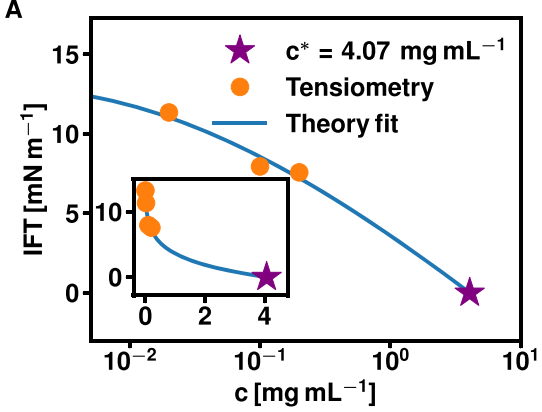

C

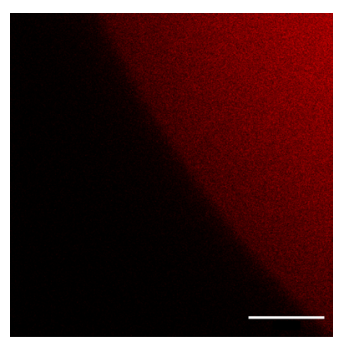

B
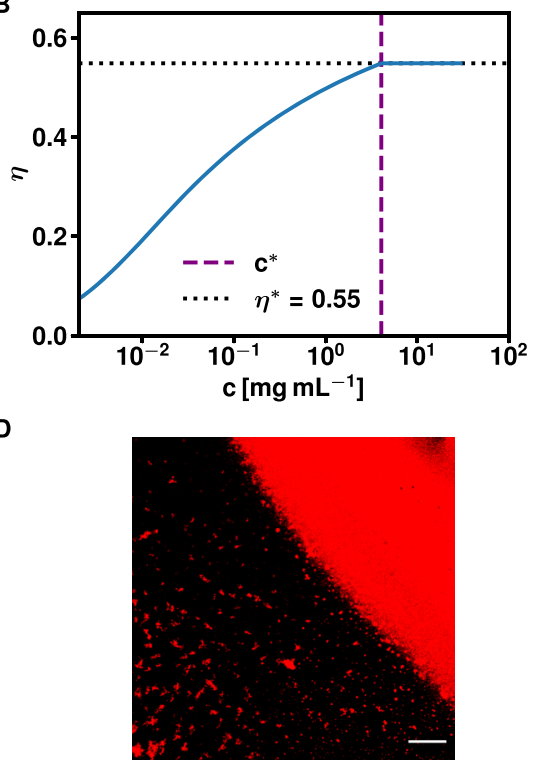

FIG. 2. Ascertaining the microemulsification concentration. (a) IFT of the silicone-water interface as a function of Noria concentration, $c$, obtained from pendant drop tensiometry, along with a fit of the theory to the data. Extrapolation of the fitted curve predicts a microemulsification concentration of $c^{\star}=4.07 \mathrm{mg} \mathrm{mL}^{-1}$. Inset: IFT as a function of $c$ on a linear scale. (b) Packing fraction at the interface, $\eta$, as a function of Noria concentration, $c$. The fit predicts a critical packing fraction $\eta^{*}=0.55$. (c) Confocal microscopy image of a stable aqueous droplet containing hydrophilic Nile blue dye without any Noria, in $5 \%$ cSt silicone oil with $20 \%$ w/w PDMS$\mathrm{NH}_{2}$. (d) Introducing $5 \mathrm{mg} \mathrm{mL}^{-1}$ of Noria results in the spontaneous formation of aqueous domains that escape into the bulk. The scale bars represent $50 \mu \mathrm{m}$. 


\section{B. Experiments}

One strategy that has successfully imparted high binding energy to a variety of nanoparticles makes use of complementary polymerligand interactions to form in situ nanoparticle surfactants at the interface. ${ }^{15}$ Using the thermodynamic analysis above, together with measurements of the IFT, we show that this enhancement is consistent with an increased binding energy, $U$, sufficient to induce microemulsification at modest volume fractions, $\phi^{*}$.

By suspending ligand-coated nanoparticles in one phase, and dissolving a favorably interacting polymer in the other, binding occurs when the two meet at the interface [Fig. 1(a)]. These nanoparticle surfactant assemblies have been shown to form monolayers at the liquid-liquid interface using grazing incidence $\mathrm{x}$-ray scattering, ${ }^{38}$ atomic force microscopy, ${ }^{39}$ and $\mathrm{x}$-ray photon correlation spectroscopy. ${ }^{40}$ Here, we use carboxy-functionalized Noria, a nanoporous organic cage, ${ }^{41,42}$ chosen for its high charge density. At the oil-water interface, the carboxy groups on the Noria form ammonium-carboxylate complexes with amine-terminated polydimethylsiloxane (PDMS- $\mathrm{NH}_{2}$ ) dissolved at $20 \% \mathrm{w} / \mathrm{w}$ in $5 \% \mathrm{cSt}$ silicone oil. In all experiments, $\mathrm{pH}=5$ was maintained using a $5 \mathrm{mM}$ MES buffer. The low $\mathrm{pH}$ of the aqueous phase served to reduce the interfacial tension of the oil-water-PDMS- $\mathrm{NH}_{2}$ system in the absence of nanoparticles by protonation of the amine groups of PDMS- $\mathrm{NH}_{2},{ }^{43}$ and it also served to increase the binding energy of the nanoparticles. ${ }^{44}$ In the traditional language of microemulsions, the PDMS- $\mathrm{NH}_{2}$ surfactant markedly reduces the oil-water interfacial tension, while the Noria (not interfacially active by itself) acts as a co-surfactant that drives the oil-water system to spontaneously increase the surface area.

We measured the IFT of this system using pendant drop tensiometry for Noria concentrations ranging from $0 \mathrm{mg} \mathrm{mL}^{-1}$ to $0.2 \mathrm{mg} \mathrm{mL} \mathrm{m}^{-1}$, and a fit of Eqs. (1) and (2) was performed using the binding strength, $U$, and particle radius, $r$, as free parameters
[Fig. 2(a)]. Our fit estimates that the radius of the Noria is $r=0.51 \mathrm{~nm}$, very close to X-ray diffraction results, ${ }^{42}$ and that the binding strength is $U=11 k_{\mathrm{B}} T$, which supports the hypothesis that particles adsorb reversibly to the interface. The estimated binding energy is four times larger than the prediction of the Pieranski model ${ }^{35}$ [Fig. 1(d)] and might be due to the ability of all of the carboxy groups on the surface of the Noria to interact with PDMS- $\mathrm{NH}_{2}$, even though the Noria is partly in the aqueous phase. Extrapolation of the fit predicts that spontaneous emulsification occurs at a concentration of $c^{*}=4.07 \mathrm{mg} \mathrm{mL}^{-1}$, corresponding to a volume fraction $\phi^{*}=0.0023$, and packing fraction $\eta^{*}=0.55$ [Fig. 2(b)].

This prediction is validated by direct observation of spontaneous emulsification using confocal microscopy performed on aqueous droplets containing $1 \% \mathrm{w} / \mathrm{w}$ Nile blue dye in the presence and absence of Noria [Figs. 2(c) and 2(d), respectively], immersed in PDMS $-\mathrm{NH}_{2}$ containing silicone oil. Since the dye is hydrophilic, aqueous regions of the sample appear bright red. In the absence of Noria, the surface of the water droplet is stable and smooth, but for a Noria concentration above $c^{*}$, specifically $5 \mathrm{mg} \mathrm{mL}^{-1}$, we observe the spontaneous generation of aqueous droplets that pinch off the liquid-liquid interface and swell as they escape into bulk.

To probe the microemulsified state, we extend our model to allow an aqueous volume, $V$, to be arranged in $m \propto 6 V D^{-3}$ identical droplets with the diameter $D$ and total surface area $A=6 V D^{-1}$ [Fig. 3(a)]. ${ }^{30}$ As a result, the total free energy becomes

$$
\begin{aligned}
F_{\text {Tot }}(\eta, D)= & F_{2 \mathrm{~d}}(\eta, A(D))+F_{3 \mathrm{~d}}\left(\phi-\frac{8 \eta r}{D}\right) \\
& +A(D)\left[\gamma-\frac{\eta}{\pi r^{2}}\left[U+k_{\mathrm{B}} T \ln \frac{2 r}{\Lambda}\right]\right] \\
& +k_{\mathrm{B}} \operatorname{Tm}(D)[\ln m(D)-1] .
\end{aligned}
$$

A

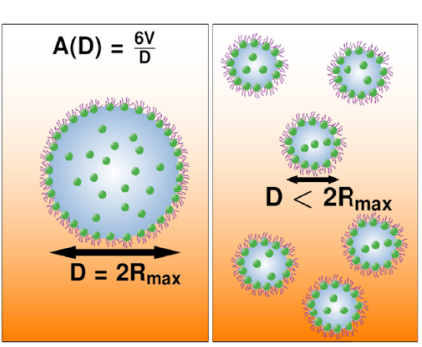

C

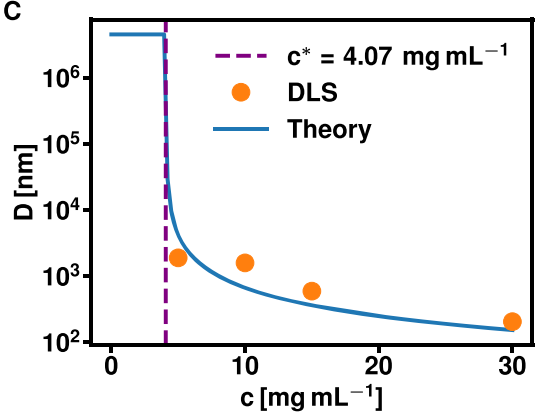

B

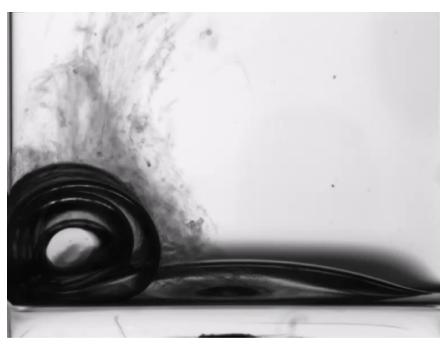

D

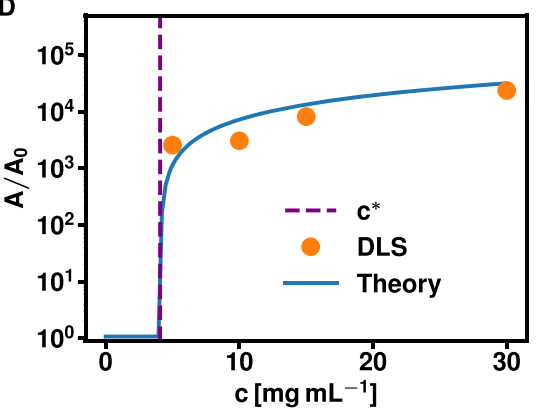

FIG. 3. Spontaneous emulsification regime. (a) Schematic illustration of the thermodynamic states described by Eq. 3. (b) Macroscopic observation of spontaneous emulsification obtained using video microscopy. Droplets containing $15 \mathrm{mg} \mathrm{mL}^{-1}$ of Noria produce a haze of aqueous compartments over a period of $12 \mathrm{~h}$, as can be seen from the videos of the supplementary material. (c) Microemulsion droplet diameter, $D$, as determined by dynamic light scattering and our model's prediction, as a function of the Noria concentration, $c$. (d) Estimated increase in the exposed oil-water surface area, as inferred from the droplet volume, $V=50 \mu \mathrm{l}$, and from $D$. 
Here, $F_{2 \mathrm{~d}}$ and $F_{3 \mathrm{~d}}$ are the Helmholtz free energies corresponding to the chemical potentials $\mu_{2 \mathrm{~d}}$ and $\mu_{3 \mathrm{~d}}$. The thermodynamically stable state to which an oversaturated droplet relaxes [Fig. 3(b)] is obtained by minimizing the free energy in Eq. (3) with respect to the packing fraction, $\eta$, and microemulsion droplet diameter, $D$, which leads to the following equations that must be satisfied:

$$
\begin{gathered}
\mu_{2 \mathrm{~d}}(\eta)-U-k_{\mathrm{B}} T \ln \frac{2 r}{\Lambda}=\mu_{3 \mathrm{~d}}\left(\phi-\frac{8 \eta r}{D}\right), \\
\gamma-k_{\mathrm{B}} T \frac{\eta Z(\eta)}{\pi r^{2}}=\frac{3 k_{\mathrm{B}} T}{\pi D^{2}} \ln \frac{\pi D^{3}}{6 V} .
\end{gathered}
$$

Using the fit parameters obtained from the IFT measurements [Fig. 2(a)], we obtain predictions for $\eta$ [Figs. 2(b)] and $D$, as a function of the Noria concentration, which show good agreement with dynamic light scattering measurements of $50 \mu \mathrm{l}$ aqueous droplets [Fig. 3(c)], from which we infer an increase of up to four orders of magnitude of the oil-water interfacial area [Fig. 3(d)] as the Noria concentration increases.

\section{DISCUSSION AND CONCLUSION}

Previous experimental $^{45,46}$ and theoretical ${ }^{24,47}$ studies of molecular microemulsification show that it is a continuous, second-order phase transition with the same critical exponents as the 3D Ising model. Although our theory does not take microscopic fluctuations of the interface into account, which means that it cannot deliver the correct critical exponents, the mean-field characterization of the microemulsification transition is qualitatively consistent with more detailed measurements and models. This model's description of the microemulsification transition is obtained by inserting the material parameters of our system and the equilibrium values of $\eta$ and $D$ into Eq. (3) to obtain the Helmholtz free energy, $F_{\min }(\phi, V, \gamma, U, r)$ [Fig. 4(a)]. Numerical differentiation reveals a kink in the first derivative of the free energy at the microemulsification concentration $c^{*}$ [Fig. 4(b)]. This kink is due to a discontinuity in the second derivative of the free energy that becomes sharper as the droplet volume goes to infinity [Fig. 4(c)], which indicates that the microemulsion transition is a continuous, second-order phase transition in accordance with the aforementioned studies. Further support of this conclusion is provided in the supplementary material, where we derive a complementary lattice model with a more accurate critical behavior. Our results are summarized in the microemulsion phase diagram depicted in Fig. 4(d), which delineates the microemulsified from the phase separated state as a function of the thermodynamic drive for particle adsorption, $\beta \mu_{3 \mathrm{~d}}(\phi)+\beta U$, and the free energy reduction due to an adsorbed particle's screening of the oil-water interface, $\beta \gamma \pi r^{2}$.

Further studies on the nature of nanoparticle-induced microemulsification include determining which macroscopic emulsion phases are stable, ${ }^{48}$ determining the extent to which varying ionic strength shifts the critical concentration, and an exploration of how the nanoparticle size affects microemulsification. One corollary of this framework is that the phase transitions that interfacially active materials undergo will have a recognizable signature in the IFT vs concentration curves due to the intimate relationship between the equation of state and the IFT, as shown in the supplementary material.

Using a combined theoretical and experimental approach, we have demonstrated that sufficiently small and interfacially active nanoparticles are capable of destabilizing an oil-water interface to spontaneously create a stable emulsion over observable timescales and without mechanical agitation. Furthermore, the fitting procedure used herein successfully retrieves microscopic information
A

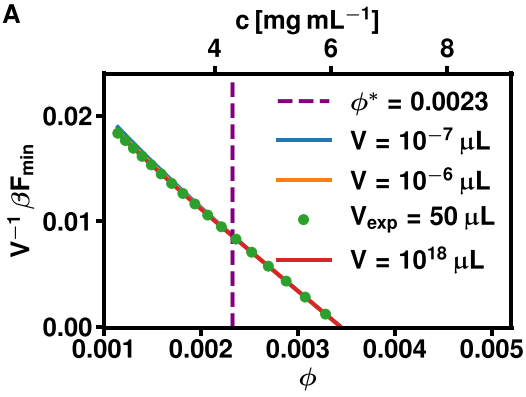

C

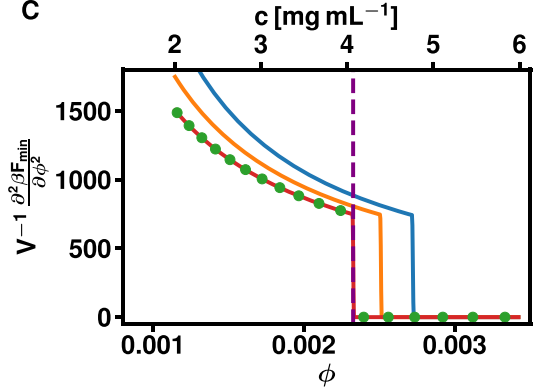

B

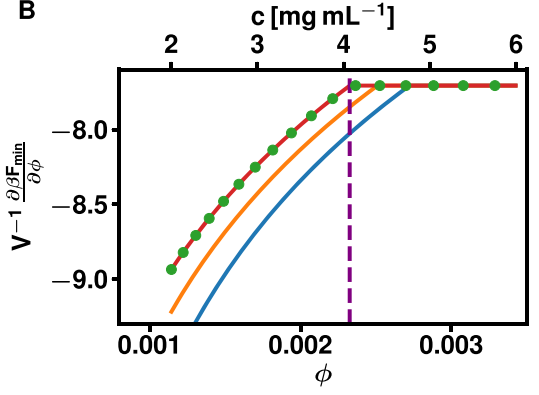

D

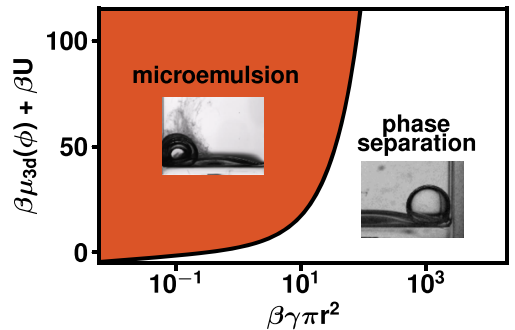

FIG. 4. Phase diagram for spontaneous emulsification. (a) Reduced Helmholtz free energy, $V^{-1} \beta F_{\min }$, as a function of the volume fraction of the nanoparticle surfactant, $\phi$, for volumes ranging from $10^{-17} \mu \mathrm{l}$ to $10^{18} \mu \mathrm{l}$ of the aqueous phase. The green circles correspond to the volume used in the experiment and are numerically indistinguishable from the thermodynamic limit. (b) First derivative of the free energy with respect to $\phi$ [colors as in (a)]. (c) The discontinuity in the second derivative of the Helmholtz free energy is the model's prediction that microemulsification is a second-order phase transition [colors as in (a)]. (d) Phase diagram delineating the phase separated and microemulsified states as a function of $\beta \gamma \pi r^{2}$ and $\beta \mu_{3 d}(\phi)+\beta U$. 
from macroscopic tensiometry experiments and accurately predicts critical concentrations and droplet sizes across several decades of length scales and concentrations. In addition to constituting a broad new class of microemulsifiers, particle-based microemulsions are expected to be robust to temperature changes ${ }^{49}$ and provide the possibility of exposing enormous interfacial area to nanoparticles with plasmonic, catalytic, or biological functionality, leading to the self-assembly of all-liquid sensing devices, chemical scavengers, and liquid microscale and nanoscale reaction vessels.

\section{MATERIALS AND METHODS}

In all experiments, we used carboxy terminated Noria nanoparticles $^{50}$ with an approximate molar mass of $M=1700 \mathrm{~g} \mathrm{~mol}^{-1}$, dispersed in water with $\mathrm{pH}=5$, using a $5 \mathrm{mM}$ MES buffer, and a $5 \%$ cSt silicone oil phase containing $20 \% \mathrm{w} / \mathrm{w}$ PDMS- $\mathrm{NH}_{2}$. Interfacial tensions were measured using the pendant drop method and, absent of Noria, the silicone-water interfacial tension after $1 \mathrm{~h}$ was $13.3 \mathrm{mN} \mathrm{m}^{-1}$. Introducing Noria to the aqueous phase led to the association of multiple polymer ligands with the carboxy groups of the Noria. Interfacial tension measurements were conducted with Noria concentrations of $0.02 \mathrm{mg} \mathrm{mL}^{-1}, 0.1 \mathrm{mg} \mathrm{mL}^{-1}$, and $0.2 \mathrm{mg}$ $\mathrm{mL}^{-1}$, which resulted in effective surface tension measurements of $11.4 \mathrm{mN} \mathrm{m}^{-1}, 7.96 \mathrm{mN} \mathrm{m}^{-1}$, and $7.6 \mathrm{mN} \mathrm{m}^{-1}$. For Noria concentrations above $5 \mathrm{mg} \mathrm{mL}^{-1}$, spontaneous emulsification occurred.

The time evolution of a microemulsifying droplet surface was studied using a confocal laser scanning microscope with wavelength $637 \mathrm{~nm}$. To image the aqueous part of the system, $0.1 \%$ Nile blue dye was added to a $25 \mu \mathrm{l}$ water droplet, which was deposited in silicone oil containing PDMS- $\mathrm{NH}_{2}$.

Microemulsions droplet sizes were measured using dynamic light scattering with a Malvern Zetasizer, He-Ne laser, set to $633 \mathrm{~nm}$. $50 \mu \mathrm{l}$ aqueous droplets containing Noria at concentrations of $5 \mathrm{mg} \mathrm{mL}^{-1}, 10 \mathrm{mg} \mathrm{mL}^{-1}, 15 \mathrm{mg} \mathrm{mL}^{-1}$, and $30 \mathrm{mg} \mathrm{mL}^{-1}$ were deposited into a silicone oil bath, and an average droplet size of $1902 \mathrm{~nm}, 1600 \mathrm{~nm}, 596 \mathrm{~nm}$, and $206 \mathrm{~nm}$ was measured. Close to the critical concentration, microemulsion diameters were observed that were of similar size as in the previous work on AOT, water, and n-decane systems.

Fits to experimental data were performed using the fitting algorithms in the supplementary material. The code is written in Python and makes use of the numpy and matplotlib libraries.

\section{SUPPLEMENTARY MATERIAL}

See the supplementary material for detailed derivations and videos V1 and V2, along with experimental data and data analysis scripts.

\section{AUTHORS' CONTRIBUTIONS}

J.H., L.A., and P.L.G. developed the theoretical model. Experimental measurements were designed by Y.J., H.H, J.F., P.D.A., B.A.H., and T.P.R. and were conducted by Y.J. and H.H. The nanoparticles were synthesized by J.Y. and B.A.H. This manuscript was written by J.H., T.P.R., and P.L.G. J.H., Y.J., and H.H. contributed equally to this work.

\section{ACKNOWLEDGMENTS}

This work was supported by the U.S. Department of Energy, Office of Science, Office of Basic Energy Sciences, Materials Sciences and Engineering Division, under Contract No. DE-AC0205-CH11231 within the Adaptive Interfacial Assemblies towards Structuring Liquids program (Grant No. KCTR16). The authors gratefully acknowledge Pratima Satish and Georg Menzl for helpful discussions and insights.

\section{DATA AVAILABILITY}

The data that support the findings of this study are available within this article and its supplementary material.

\section{REFERENCES}

${ }^{1}$ Y. Chevalier and M.-A. Bolzinger, Colloids Surf,, A 439, 23 (2013).

${ }^{2}$ H.-F. Eicke and J. Rehak, Helv. Chim. Acta 59, 2883 (1976).

${ }^{3}$ P. G. De Gennes and C. Taupin, J. Phys. Chem. 86, 2294 (1982).

${ }^{4}$ A. Graciaa, J. Lachaise, J. G. Sayous, P. Grenier, S. Yiv, R. S. Schechter, and W. H. Wade, J. Colloid Interface Sci. 93, 474 (1983).

${ }^{5}$ J. C. Löpez-Montilla, P. E. Herrera-Morales, S. Pandey, and D. Shah, J. Dispersion Sci. Technol. 23, 219 (2002).

${ }^{6}$ D. J. McClements, Soft Matter 8, 1719 (2012).

${ }^{7}$ C. Solans, D. Morales, and M. Homs, Curr. Opin. Colloid Interface Sci. 22, 88 (2016).

${ }^{8}$ M. J. Lawrence and G. D. Rees, Adv. Drug Delivery Rev. 45, 89 (2000).

${ }^{9}$ H. Shao, N. Xi, and Y. Zhang, Sci. Rep. 8, 10565 (2018).

${ }^{10}$ C. van der Wel, R. K. Bhan, R. W. Verweij, H. C. Frijters, Z. Gong, A. D. Hollingsworth, S. Sacanna, and D. J. Kraft, Langmuir 33, 8174 (2017).

${ }^{11}$ M. Schwarze, T. Pogrzeba, I. Volovych, and R. Schomäcker, Catal. Sci. Technol. 5, 24 (2015).

${ }^{12}$ P.-P. Fang, S. Chen, H. Deng, M. D. Scanlon, F. Gumy, H. J. Lee, D. Momotenko, V. Amstutz, F. Cortés-Salazar, C. M. Pereira, Z. Yang, and H. H. Girault, ACS Nano 7, 9241 (2013).

${ }^{13}$ X. Liu, N. Kent, A. Ceballos, R. Streubel, Y. Jiang, Y. Chai, P. Y. Kim, J. Forth, F. Hellman, S. Shi, D. Wang, B. A. Helms, P. D. Ashby, P. Fischer, and T. P. Russell, Science 365, 264 (2019).

${ }^{14}$ C. Huang, Z. Sun, M. Cui, F. Liu, B. A. Helms, and T. P. Russell, Adv. Mater. 28, 6612 (2016).

${ }^{15}$ S. Shi and T. P. Russell, J. Adv. Mater. 30, 1800714 (2018).

${ }^{16}$ K. Stratford, R. Adhikari, I. Pagonabarraga, J.-C. Desplat, and M. E. Cates, Science 309, 2198 (2005).

${ }^{17}$ M. Reeves, A. T. Brown, A. B. Schofield, M. E. Cates, and J. H. J. Thijssen, Phys. Rev. E 92, 032308 (2015).

${ }^{18}$ S. Sacanna, W. K. Kegel, and A. P. Philipse, Phys. Rev. Lett. 98, 158301 (2007).

${ }^{19}$ D. J. Kraft, J. W. J. de Folter, B. Luigjes, S. I. R. Castillo, S. Sacanna, A. P. Philipse, and W. K. Kegel, J. Phys. Chem. B 114, 10347 (2010).

${ }^{20}$ W. K. Kegel and J. Groenewold, Phys. Rev. E 80, 030401 (2009).

${ }^{21}$ J. Zwanikken, K. Ioannidou, D. Kraft, and R. van Roij, Soft Matter 7, 11093 (2011).

${ }^{22}$ L. M. Prince, J. Colloid Interface Sci. 23, 165 (1967).

${ }^{23}$ S. A. Safran and L. A. Turkevich, Phys. Rev. Lett. 50, 1930 (1983).

${ }^{24}$ B. Widom, J. Chem. Phys. 84, 6943 (1986).

${ }^{25}$ S. Puvvada and D. Blankschtein, J. Chem. Phys. 92, 3710 (1990).

${ }^{26}$ E. Ruckenstein, J. Colloid Interface Sci. 204, 143 (1998).

${ }^{27}$ R. Nagarajan and E. Ruckenstein, Langmuir 16, 6400 (2000).

${ }^{28}$ C. A. Bearchell and D. M. Heyes, Phys. Chem. Chem. Phys. 3, 5255 (2001).

${ }^{29}$ G. Kaptay, Langmuir 31, 5796 (2015). 
${ }^{30}$ G. Kaptay, Langmuir 33, 10550 (2017)

${ }^{31}$ J. A. Barker and D. Henderson, J. Chem. Phys. 47, 4714 (1967).

${ }^{32}$ J. Hasnain, G. Menzl, S. Jungblut, and C. Dellago, Soft Matter 13, 930 (2017).

${ }^{33}$ J. D. Weeks, D. Chandler, and H. C. Andersen, J. Chem. Phys. 54, 5237 (1971).

${ }^{34}$ A. Santos, M. López de Haro, and S. Bravo Yuste, J. Chem. Phys. 103, 4622 (1995).

${ }^{35}$ P. Pieranski, Phys. Rev. Lett. 45, 569 (1980).

${ }^{36}$ K. Schwenke, L. Isa, and E. Del Gado, Langmuir 30, 3069 (2014).

${ }^{37}$ H. Hou, J. Li, X. Li, J. Forth, J. Yin, X. Jiang, B. A. Helms, and T. P. Russell, Angew. Chem., Int. Ed. 58, 10142 (2019).

${ }^{38}$ M. Cui, T. Emrick, and T. P. Russell, Science 342, 460 (2013).

${ }^{39}$ Y. Chai, J. Hasnain, K. Bahl, M. Wong, D. Li, P. L. Geissler, P. Y. Kim, Y. Jiang, P. Gu, S. Li, D. Lei, B. A. Helms, T. P. Russell, and P. D. Ashby, Sci. Adv. 6(48), eabb8675 (2020).

${ }^{40}$ M. Cui, C. Miesch, I. Kosif, H. Nie, P. Y. Kim, H. Kim, T. Emrick, and T. P. Russell, Nano Lett. 17, 6855 (2017).
${ }^{41}$ H. Kudo, R. Hayashi, K. Mitani, T. Yokozawa, N. C. Kasuga, and T. Nishikubo, Angew. Chem., Int. Ed. 45, 7948 (2006).

${ }^{42}$ R. S. Patil, D. Banerjee, C. M. Simon, J. L. Atwood, and P. K. Thallapally, Chem.: Eur. J. 22, 12618 (2016)

${ }^{43}$ C. Huang, M. Cui, Z. Sun, F. Liu, B. A. Helms, and T. P. Russell, Langmuir 33, 7994 (2017).

${ }^{44}$ Y. Chai, A. Lukito, Y. Jiang, P. D. Ashby, and T. P. Russell, Nano Lett. 17, 6453 (2017).

${ }^{45}$ J. S. Huang and M. W. Kim, Phys. Rev. Lett. 47, 1462 (1981).

${ }^{46}$ S. H. Chen, J. Rouch, and P. Tartaglia, Physica A 204, 134 (1994).

${ }^{47}$ J. C. Wheeler, Annu. Rev. Phys. Chem. 28, 411 (1977).

${ }^{48}$ D. Langevin, D. Guest, and J. Meunier, Colloids Surf. 19, 159 (1986).

${ }^{49}$ S. Tcholakova, Z. Valkova, D. Cholakova, Z. Vinarov, I. Lesov, N. Denkov, and S. K. Smoukov, Nat. Commun. 8, 15012 (2017).

${ }^{50}$ H. Kudo, D. Watanabe, T. Nishikubo, K. Maruyama, D. Shimizu, T. Kai, T. Shimokawa, and C. K. Ober, J. Mater. Chem. 18, 3588 (2008). 